OPEN ACCESS

Edited by:

Rosalba Giugno,

University of Verona, Italy

Reviewed by:

Alfonso Urso,

Institute for High Performance Computing and Networking (ICAR),

Luciano Cascione

Istituto Oncologico della Svizzera

Italiana, Switzerland

*Correspondence:

Fei Hua

huafei1970@suda.edu.cn

Xiaoyu Yang

jsczyxy@163.com

tThese authors have contributed equally to this work

Specialty section: This article was submitted to Bioinformatics and

Computational Biology, a section of the journal

Frontiers in Genetics

Received: 15 March 2019 Accepted: 21 October 2019 Published: 15 November 2019

Citation:

Tao L, Yang L, Huang X, Hua F and Yang $X$ (2019) Reconstruction

and Analysis of the IncRNA-

miRNA-mRNA Network Based

on Competitive Endogenous RNA

Reveal Functional IncRNAs in Dilated

Cardiomyopathy.

Front. Genet. 10:1149.

doi: 10.3389/fgene.2019.01149

\section{Reconstruction and Analysis of the IncRNA-miRNA-mRNA Network Based on Competitive Endogenous RNA Reveal Functional IncRNAs in Dilated Cardiomyopathy}

\author{
Lichan Tao ${ }^{1 \dagger}$, Ling Yang ${ }^{1 \dagger}$, Xiaoli Huang ${ }^{2 \dagger}$, Fei Hua ${ }^{2 *}$ and Xiaoyu Yang ${ }^{1 *}$ \\ ${ }^{1}$ Department of Cardiology, The Third Affiliated Hospital of Soochow University, Changzhou, China, ${ }^{2}$ Department of \\ Endocrinology, The Third Affiliated Hospital of Soochow University, Changzhou, China
}

Dilated cardiomyopathy (DCM) is an important cause of sudden death and heart failure with an unknown etiology. Recent studies have suggested that long non-coding RNA (IncRNA) can interact with microRNA (miRNA) and indirectly interact with mRNA through competitive endogenous RNA (ceRNA) activities. However, the mechanism of ceRNA in DCM remains unclear. In this study, a miRNA array was first performed using heart samples from DCM patients and healthy controls. For further validation, we conducted real-time quantitative reverse transcription (RT)-PCR using samples from DCM patients and a doxorubicin-induced rodent model of cardiomyopathy, revealing that miR-144-3p and miR-451a were down-regulated, and miR-21-5p was up-regulated. Based on the ceRNA theory, we constructed a global triple network using data from the National Center for Biotechnology Information Gene Expression Omnibus (NCBI-GEO) and our miRNA array. The IncRNA-miRNA-mRNA network comprised 22 IncRNA nodes, 32 mRNA nodes, and 11 miRNA nodes. Hub nodes and the number of relationship pairs were then analyzed, and the results showed that two IncRNAs (NONHSAT001691 and NONHSAT006358) targeting miR-144/451 were highly related to DCM. Then, cluster module and random walk with restart for the ceRNA network were analyzed and identified four IncRNAs (NONHSAT026953/NONHSAT006250/NONHSAT133928/NONHSAT041662) targeting miR-21 that were significantly related to DCM. This study provides a new strategy for research on DCM or other diseases. Furthermore, IncRNA-miRNA pairs may be regarded as candidate diagnostic biomarkers or potential therapeutic targets of DCM.

Keywords: long non-coding RNA, microRNA, competitive endogenous RNA, IncRNA-miRNA-mRNA network, dilated cardiomyopathy

\section{INTRODUCTION}

Chronic heart failure (CHF), a main cause of morbidity and mortality, especially in aging. CHF is a complex clinical syndrome resulting from various structural and functional impairments in ventricular filling or blood ejection (Garin et al., 2014). The lifetime risk of developing CHF has been calculated to range from 20 to $33 \%$ worldwide, and nearly half of the patients with CHF will 
die within 5 years despite all the advanced therapies (Dobre et al., 2014). In addition, as the population ages, the cost associated with $\mathrm{CHF}$ is also expected to increase substantially. The etiology of CHF can be classified as ischemic (ICM) or non-ischemic cardiomyopathy (NICM), and dilated cardiomyopathy (DCM) is one of the major causes of ICM. In contrast to revascularization therapies for ICM, novel treatments for DCM remain scarce. Therefore, studies focused on developing new strategies for DCM are urgently required.

Accumulating evidence has suggested that rather than being transcriptional noise, diverse non-coding RNAs (ncRNAs) serve as master regulators in $\mathrm{CHF}$ initiation and progression at the post-transcriptional level (Kumarswamy and Thum, 2013; Pinet and Bauters, 2015; Thum, 2015). Among them, long non-coding RNAs (lncRNAs) are conventionally described as transcripts longer than 200 nucleotides with no or little protein-coding capacity (Greco et al., 2016; Dangwal et al., 2017). Owing to their versatility, lncRNAs have been reported to participate in several cellular processes ranging from chromatin modification and RNA stability to translational control. Biochemically, lncRNAs exert their function via RNARNA, RNA-DNA, or RNA-protein interactions (Li et al., 2013; Dey et al., 2014; Shi et al., 2015). Of note, lncRNAs have been reported to competitively interact with microRNAs (miRNAs) and thus inhibit target mRNA degradation by a competitive endogenous RNA (ceRNA) regulatory mechanism (Sen et al., 2014; Tay et al., 2014).

Recently, studies have identified several aberrantly expressed lncRNAs in CHF models. Moreover, overexpression/ knockdown of specific lncRNAs have been reported to significantly influence the process of cardiac development, aging, hypertrophy, and fibrosis in mice (Cheng et al., 2014; Michalik et al., 2014; Devaux et al., 2015; Pourrajab et al., 2015; Uchida and Dimmeler, 2015). However, because of low sequence conservation among species, it is difficult to extend the findings derived from murine models to humans; therefore, little is known about the function of lncRNAs in human hearts.
Current reports of lncRNAs in DCM patients are focused on expression profiles from RNA sequencing or microarray (Schiano et al., 2017; Haas et al., 2018). Therefore, considering the large number and limited knowledge of lncRNAs, how to develop computational model for identification of lncRNAs and downstream miRNAs or mRNAs are of significant importance.

In our study, we conducted a microarray profile of miRNAs in myocardial biopsy samples from end-stage DCM patients compared with those in normal myocardial samples. Furthermore, based on the ceRNA theory, we constructed a global triple network by using data from the GEO database, as lncRNA and mRNA form a triplet by sharing the same miRNA. We identified human DCM-related lncRNAs with high reliability and our results showed that the lncRNA-miRNA-mRNA network provides a new understanding of the mechanisms and potential therapeutic targets for DCM.

\section{MATERIALS AND METHODS}

\section{Patients and Tissue Samples}

The experimental procedure for evaluating the differential expression of lncRNA/miRNA/mRNA is described in (Figure 1) The study protocol was approved by the Medical Ethics Committee of the Third Affiliated Hospital of Soochow University in Changzhou, Jiangsu Province, China, and informed consent was obtained from each patient. Tissues for detection were collected from the left ventricular wall of explanted hearts from patients with a diagnosis of DCM undergoing heart transplantation (clinical data were presented in our previous paper) (Tao et al., 2016) and from unmatched healthy donors.

\section{Animal Model}

Doxorubicin-induced cardiomyopathy mouse model was induced by chronically administrating mice with either doxorubicin or phosphate-buffered saline (PBS) by six intraperitoneal (i.p)

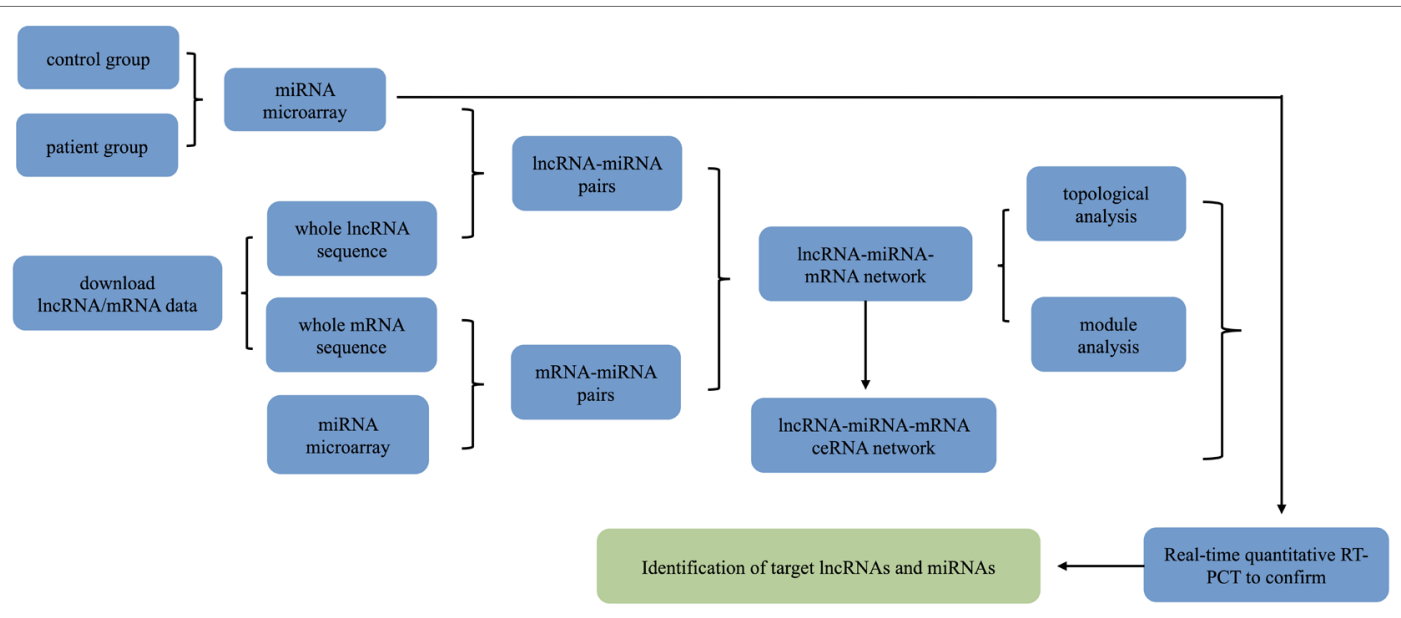

FIGURE 1 | Schematic depiction of the experimental design and flowchart of the steps performed in this study. 
injections (day 0, 2, 4, 6, 8, and 10) at a dose of $4 \mathrm{mg} / \mathrm{kg}$. After 4 weeks, echocardiography was performed and mice were sacrificed.

\section{RNA Isolation}

Total RNA was harvested using TRIzol and purified with the RNeasy mini kit (Qiagen, Hilden, Germany) according to manufacturer's instructions. cDNA synthesis was performed with Bio-Rad iScripTM cDNA Synthesis Kit (Bio-Rad, Hercules, CA, USA) in a reaction volume of $10 \mu \mathrm{l}$.

\section{miRNA Microarray Gene Expression Profiling}

The miRNA expression profiling assay system based on Affymetrix 4.0 (OE Biotech's, Shanghai, China) was used to perform miRNA expression profiling of myocardial samples from three patients with DCM and three healthy donors (No. GSE112556). Clinical and echocadiography parmeters for patients with dilated cardiomyopathy were presented in Supplemental Table 1. The threshold of up-regulated or down-regulated miRNA was a fold change greater than two, and $P<0.05$ using Student's t-test was considered statistically significant.

\section{IncRNA and mRNA Microarray Data}

GEO is a public functional genomics data repository that supports MIAME-compliant data submissions. Human lncRNA/ mRNA expression profiles were downloaded from NCBI-GEO (GSE42955) (Molina-Navarro et al., 2013). The threshold of up-regulated or down-regulated lncRNA/mRNA was a fold change greater than 1.5 , and $P<0.05$ using Student's t-test was considered statistically significant. This database was further analyzed with our miRNA microarray profile.

\section{Real-Time Quantitative Reverse Transcription (Rt)-PCR}

For quantitative mRNA analysis, a template equivalent to $400 \mathrm{ng}$ of total RNA was subjected to 40 cycles of quantitative PCR using the Takara SYBR Premix Ex TaqTM (TliRNaseH Plus, Takara, Tokyo, Japan) in the 7900HT Fast Real-Time PCR System. The absolute expression levels of miRNAs were normalized to the internal control small nuclear U6 and the expression levels of $\operatorname{lncRNAs}$ were normalized to $18 \mathrm{~s}$, all the data were calculated by the $\Delta \Delta \mathrm{Ct}$ method. Each reaction was primed using a genespecific stem-loop primer. The RT stem-loop primers and PCR primers are listed in Supplemental Table 2.

\section{Prediction of the miRNA Targets of IncRNAs and mRNAs}

The miRNA targets of lncRNA were predicted and the minimum free energy (MFE) of miRNA-lncRNA duplexes was calculated by using the RNAhybrid program. MiRNA sequences were obtained from miRbase and lncRNA sequences were obtained from NCBInucleotide. MiRNA target binding sites on the entire lncRNA sequence were predicted. Data of miRNA-mRNA interactions were downloaded from the Miranda and Targetscan prediction tools.

\section{Construction of the IncRNA-miRNA-mRNA Network}

The IncRNA-miRNA-mRNA network was constructed based on ceRNA theory as follows: (1) Expression correlation between lncRNAs and mRNAs was calculated using the Pearson correlation coefficient (PCC). The lncRNA-mRNA pairs with PCC $>0.99$ and $P$ value $<0.05$ were selected as target pairs. (2) Among the selected lncRNA-mRNA pairs, if both the lncRNA and mRNA targeted and were negatively co-expressed with a common miRNA, this lncRNA-miRNA-mRNA group was identified as a co-expression competing triplet. (3) The lncRNA-miRNA-mRNA network was visualized by using Cytoscape software.

\section{Functional Enrichment Analysis}

Significant progress in data mining has provided a wide range of bioinformatics analysis tools, including the gene ontology (GO) and Kyoto Encyclopedia of Genes and Genomes (KEGG) databases. The GO database provides gene ontologies, the annotations of genes, and gene products to terms. The combination of ontologies and annotations renders the GO database as a powerful tool for further analysis. The KEGG database is a relational database comprising searchable molecular interaction pathways and reaction networks in metabolism, various cellular processes, and multiple human diseases.

\section{Reconstruction of the Key IncRNA- miRNA-mRNA Subnetwork}

Every lncRNA and its related miRNAs and mRNAs in the global triple network were extracted to construct a new subnetwork using Cytoscape software. The number of related lncRNAmiRNA-mRNA triplets was calculated. By comparing the node degree of lncRNA and the number of related lncRNA-miRNA and miRNA-mRNA pairs, the target lncRNAs were identified. We then performed qRT-PCR to confirm the changes of lncRNA in samples from DCM patients and healthy controls. For further analysis, GO and KEGG enrichment analyses were performed for each of the validated lncRNAs by using their mRNA neighbors in the lncRNA-miRNA-mRNA subnetwork.

\section{Neonatal Rat Ventricular Cardiomyocyte and Fibroblast Isolation and miRNA Transfection}

All rats were purchased and raised in the Experimental Animal Center of Soochow University (Suzhou, Jiangsu Province, China). All procedures were in accordance with guidelines on the use and care of laboratory animals for biomedical research published by the National Institutes of Health (No. 85-23, revised 1996), and the experimental protocol was approved by the Animal Care and Use Committee of Soochow University. The isolation of neonatal rat ventricular cardiomyocytes (NRCM), fibroblasts (NRCF), and miRNA transfection were conducted as described in our previous paper (Tao et al., 2018).

All transfection and assays on cardiomyocytes and fibroblasts were conducted in serum free medium and low serum medium 
(1\% FBS), respectively. Cardiac fibroblasts at passage two were exposed to miRNA agomir versus negative control (50 nM), or antagomir versus negative control (100 nM) (RiboBio, Guangzhou, China) for $48 \mathrm{~h}$, and treated with $5 \mathrm{ng} / \mathrm{ml}$ or $10 \mathrm{ng} /$ ml TGF $\beta$ (Peprotech, Rocky Hill, NJ, USA) for $24 \mathrm{~h}$.

\section{Statistical Analysis}

In this study, data are expressed as the mean \pm SD. Student's t-test, Chi-square test, or one-way ANOVA followed by Bonferroni's post hoc test was used to compare the one-way layout data when appropriate. $P$ values less than 0.05 were considered to be significantly different. All analyses were performed using GraphPad Prism 5.

\section{RESULTS}

\section{Screening for Differentially Expressed IncRNAs, miRNAs, and mRNAs}

miRNA arrays were used to determine differentially expressed miRNAs (DEMis) in samples from DCM patients and healthy controls (Owing to the difficulty of obtaining human heart tissues, the DCM sample size was small). A total of 11 miRNAs were found to be dysregulated (fold change $>2.0 ; P<0.05$, Figure $2 \mathrm{~A}$ and Table $\mathbf{1}$ ). Based on the qRT-PCR analysis, we confirmed that miR-144-3p, miR-144-5p, and miR-451a were down-regulated and miR-21-5p was up-regulated in DCM heart samples (Figure 2B). To further confirm the changes in miRNA expression in DCM models, we determined the expression levels of miR-144-3p/5p, miR-451a, and miR-21-5p in a doxorubicininduced cardiomyopathy rodent model (the echocardiographic parameters for the doxorubicin-induced DCM model were presented in Supplemental Figure 1). Interestingly, miR-144-3p and miR-451a were consistently down-regulated, and miR-21-5p was up-regulated in the DCM rodent model (Figure 2C).

Human lncRNA/mRNA expression data were obtained from the GEO database (GSE42955). Considering the differences in expression level between samples, the threshold of up-regulated or down-regulated lncRNAs/mRNAs was a fold change greater than 1.5 , and $P<0.05$ using Student's t-test was regarded as statistically significant. A total of 61 lncRNAs and 172 mRNAs were selected for the following analysis.
TABLE 1 | miRNA microarray in samples of DCM patients and healthy controls.

\begin{tabular}{lccc}
\hline systematic_name & $\boldsymbol{P}$ value & Fold change & Regulation \\
\hline hsa-miR-144-3p & $2.57 \mathrm{E}-05$ & 212.8677 & down \\
hsa-miR-144-5p & $3.18 \mathrm{E}-06$ & 111.2795 & down \\
hsa-miR-16-2-3p & 0.047548 & 8.311692 & down \\
hsa-miR-21-3p & 0.032982 & 22.15763 & Up \\
hsa-miR-21-5p & 0.007706 & 2.248547 & Up \\
hsa-miR-338-3p & 0.030864 & 3.282057 & down \\
hsa-miR-363-3p & 0.006185 & 3.361872 & down \\
hsa-miR-451a & $3.08 E-04$ & 7.406941 & down \\
hsa-miR-551b-3p & 0.006586 & 5.052964 & down \\
hsa-miR-770-5p & 0.00854 & 20.36452 & Up \\
hsa-miR-9-3p & $1.55 E-05$ & 71.27749 & down \\
\hline
\end{tabular}

\section{IncRNA-miRNA-mRNA ceRNA Network}

First, we predicted IncRNA-miRNA and miRNA-mRNA pairs according to both base sequence and expression level. Considering that one miRNA may associate with several mRNAs or lncRNAs and that one lncRNA may also target several miRNAs, we analyzed whole miRNA microarray profiling. Based on the intersection elements, 199 miRNA-lncRNA pairs and 293 miRNA-mRNA pairs were identified (Figures 3A, B). Furthermore, 69 lncRNA-mRNA pairs were selected according to the ceRNA score and expression level (Figures 3C, D). Then the lncRNA-miRNA-mRNA network composed of 22 lncRNA nodes, 32 mRNA nodes, and 11 miRNA nodes were constructed (Figures 3D-F).

\section{Topological Analysis of the DCM-Related IncRNA-miRNA-mRNA Network}

As we know, hub nodes play significant roles in biological networks. We first analyzed the topological properties of the DCM-related IncRNA-miRNA-mRNA network. We calculated the degree, closeness, and betweenness of the network, and we ranked all the node topological features of the network. We listed the top 20 of each dimension. Interestingly, we found that six lncRNAs (Table 2) appeared in the list. Moreover, the number of first relationship pairs of lncRNA-miRNA and secondary relationship pairs of miRNA-mRNA was calculated (Table 3). Among the top 11 lncRNA-miRNA pairs, seven lncRNAs were identified in the ceRNA network (NONHSAT072651, NONHSAT006358, NONHSAT001691,
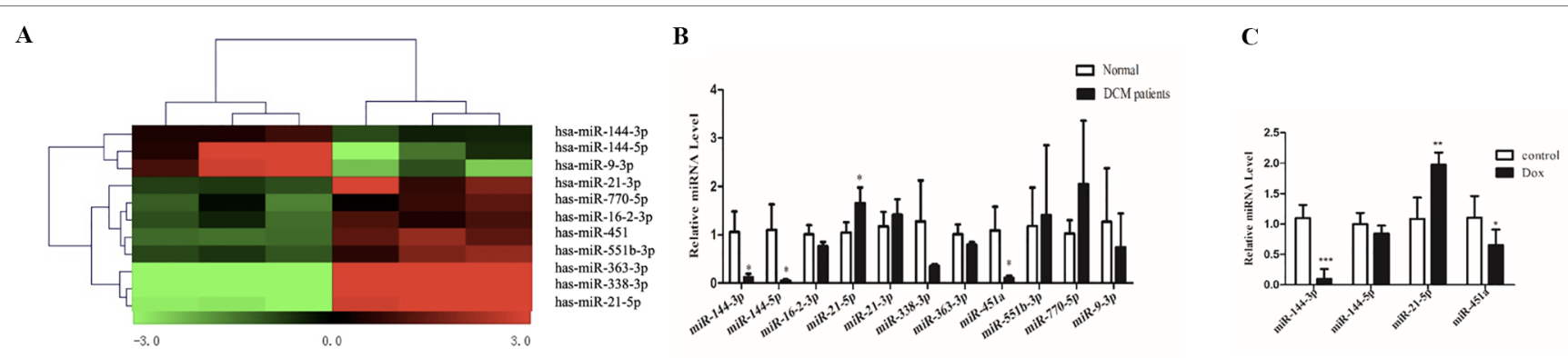

FIGURE 2 | MiRNA array using heart samples from DCM patients and healthy controls. (A). Heatmap of miRNA array between DCM patients and healthy controls and a total of 11 miRNAs were found to be dysregulated. (B). qRT-PCR analysis of miRNA between DCM patients and healthy controls ( $n=3)$. (C). qRT-PCR analysis of miRNA between doxorubicin-induced DCM models and controls $(\mathrm{n}=6)$. ${ }^{*}, p<0.05 ;{ }^{* *}, p<0.01 ;{ }^{* \star *}, p<0.001$. 


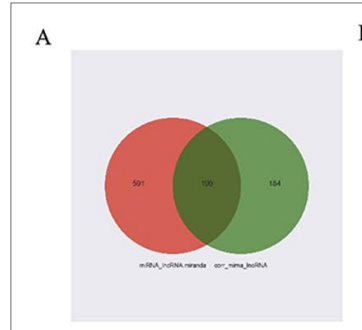

$\mathrm{E}$

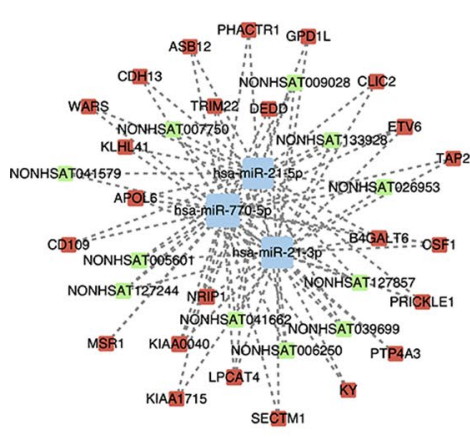

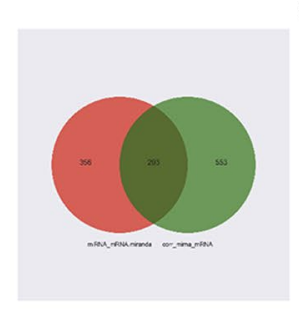

$\mathrm{F}$

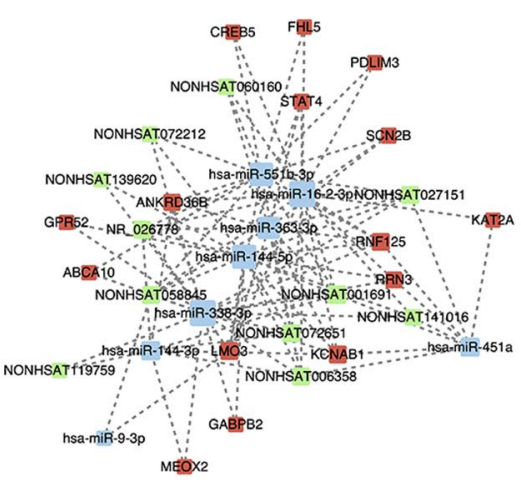

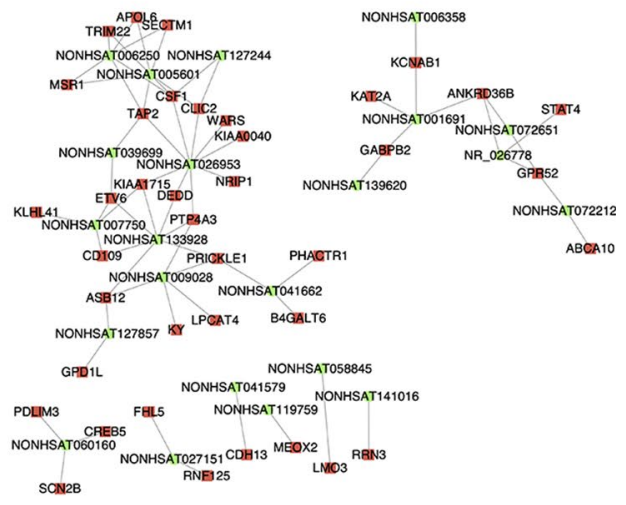

FIGURE 3 | The view of IncRNA-miRNA-mRNA network. (A-C) 199 miRNA-IncRNA pairs and 293 miRNA-mRNA pairs were identified according to both base sequence and expression level. 69 IncRNA-mRNA pairs were selected according to the ceRNA score and expression level. (D) The view of IncRNA-mRNA network. The square represents mRNA and the circle represents IncRNA. (E, F) The view of IncRNA-miRNA-mRNA network. The red represents mRNA, the green represents IncRNA, and the blue represents miRNA.

TABLE 2 | The top 20 genes in degree, betweenness and closeness.

\begin{tabular}{|c|c|c|c|c|c|}
\hline Betweenness & Closeness & Degree & Pagerank & name & Gene type \\
\hline 190.7634834 & 0.028388645 & 28 & 0.079988471 & hsa-miR-21-3p & miRNA \\
\hline 125.3606094 & 0.025448029 & 19 & 0.042194577 & hsa-miR-16-2-3p & miRNA \\
\hline 134.6788594 & 0.025448029 & 19 & 0.042922803 & hsa-miR-338-3p & miRNA \\
\hline 72.16684166 & 0.025393419 & 16 & 0.035500316 & hsa-miR-144-5p & miRNA \\
\hline 72.35483723 & 0.025375268 & 15 & 0.03428464 & hsa-miR-551b-3p & miRNA \\
\hline 13.03038694 & 0.025230988 & 8 & 0.018263656 & hsa-miR-451a & miRNA \\
\hline 18.6194442 & 0.025393419 & 7 & 0.015479801 & NONHSAT001691 & IncRNA \\
\hline 23.02997764 & 0.025375268 & 6 & 0.014139586 & LMO3 & mRNA \\
\hline 12.75746059 & 0.025375268 & 6 & 0.013537005 & KCNAB1 & mRNA \\
\hline 12.22486049 & 0.025375268 & 6 & 0.013492041 & RNF125 & mRNA \\
\hline 12.75746059 & 0.025375268 & 6 & 0.013537005 & NONHSAT006358 & IncRNA \\
\hline 9.202070258 & 0.025357143 & 5 & 0.011653333 & ANKRD36B & mRNA \\
\hline
\end{tabular}

NONHSAT027151, NONHSAT072212, NONHSAT119759, and NONHSAT139620). It is worth noting that four lncRNAs (NONHSAT001691, NONHSAT072651, NONHSAT006358, and NONHSAT027151) not only had higher betweenness and node degree but also had a higher number of lncRNA-miRNA and miRNA-mRNA pairs, which suggested that these four lncRNAs may play crucial roles in the origin and development of DCM and may be selected as key lncRNAs.

\section{Key IncRNA-miRNA-mRNA Subnetwork}

We then searched for the four key lncRNAs in the ceRNA network we had previously constructed and found that the four lncRNAs mainly targeted miR-144-3p, miR-144-5p, and miR-451. To further validate the target lncRNA, qRT-PCR was performed using samples from DCM patients and healthy controls (primers for lncRNAs are presented in Supplemental Table 2). The results suggested that NONHSAT001691 and NONHSAT006358 were significantly 
TABLE 3 | The number of IncRNA-miRNA and miRNA-mRNA pairs.

\begin{tabular}{lccc}
\hline IncRNA & $\begin{array}{c}\text { IncRNA- } \\
\text { miRNA pairs }\end{array}$ & $\begin{array}{c}\text { miRNA- } \\
\text { mRNA pairs }\end{array}$ & Total number \\
\hline NONHSAT077159 & 8 & 61 & 69 \\
NONHSAT072651 & 8 & 61 & 69 \\
NONHSAT033790 & 8 & 61 & 69 \\
NONHSAT006358 & 8 & 61 & 69 \\
NONHSAT001691 & 8 & 61 & 69 \\
NONHSAT027151 & 8 & 61 & 69 \\
NONHSAT072212 & 8 & 61 & 69 \\
NONHSAT119759 & 8 & 61 & 69 \\
NONHSAT139620 & 8 & 61 & 69 \\
NONHSAT115202 & 8 & 61 & 69 \\
NONHSAT018101 & 8 & 61 & 69
\end{tabular}

increased in DCM patients (Figure 4A). We then identified the mRNA and miRNA associated with these two lncRNAs in the global triple network and reconstructed new subnetworks. GO function and KEGG pathway annotations for each of these two lncRNAs were performed. For NONHSAT001691, we identified the biological processes of "positive regulation of cell-substrate adhesion," "positive regulation of apoptotic process" and "regulation of angiogenesis," and the enriched KEGG pathways included the "AMPK signaling pathway," PPAR signaling pathway," and "adipocytokine signaling pathway" (Figures 4B-D). For NONHSAT006358, the biological processes were similar to those of NONHSAT001691, and the KEGG pathways included the "PPAR signaling pathway," "adipocytokine signaling pathway," and "glycerophospholipid metabolism" (Figures 4E-G). The subnetworks of lncRNAs NONHSAT001691 and NONHSAT006358 are presented in Figures 4D, G.

\section{Module Analysis of the DCM-Related IncRNA-miRNA Network}

To further investigate the crosstalk between mRNAs and lncRNAs, we performed bidirectional hierarchical clustering by using $\mathrm{R}$ package "gplot." In the heat map, we discovered two modules (Figures 5A-C) that were highly related to DCM. Then, we performed GO enrichment analysis and KEGG analysis of genes in the modules (Figures 5D-G). In module 1, the "triglyceride biosynthetic process" was significantly and highly related to DCM. KEGG analysis demonstrated that "glycerophospholipid metabolism" was the most significant signaling pathway in DCM. In module 2, the "interferon gamma mediated signaling pathway" had the most notable relationship with DCM. Among all the lncRNAs in the two modules, we found 10 lncRNAs included in the ceRNA network (NONHSAT005601, NONHSAT026953, NONHSAT006250, NONHSAT007750, NONHSAT127244, NONHSAT127857, NONHSAT133928, NONHSAT009028, NONHSAT041662, and NONHSAT039699). Similar to previous approaches, we also conducted qRT-PCR using heart tissues from DCM patients and healthy controls. NONHSAT026953, NONHSAT006250, NONHSAT133928, and NONHSAT041662 were down-regulated in the DCM group. Interestingly, miR21-5p was the major target of these four lncRNAs. As miR-21-5p has been validated in DCM patients, the involvement of these four lncRNAs in DCM was further confirmed.

\section{miR-144-3p/451a Play Different Roles in NRCM and NRCF in Vitro}

Next, we determined the relative expression level of miR$144 / 451 \mathrm{a} / 21$ in isolated neonatal rat cardiac cardiomyocytes

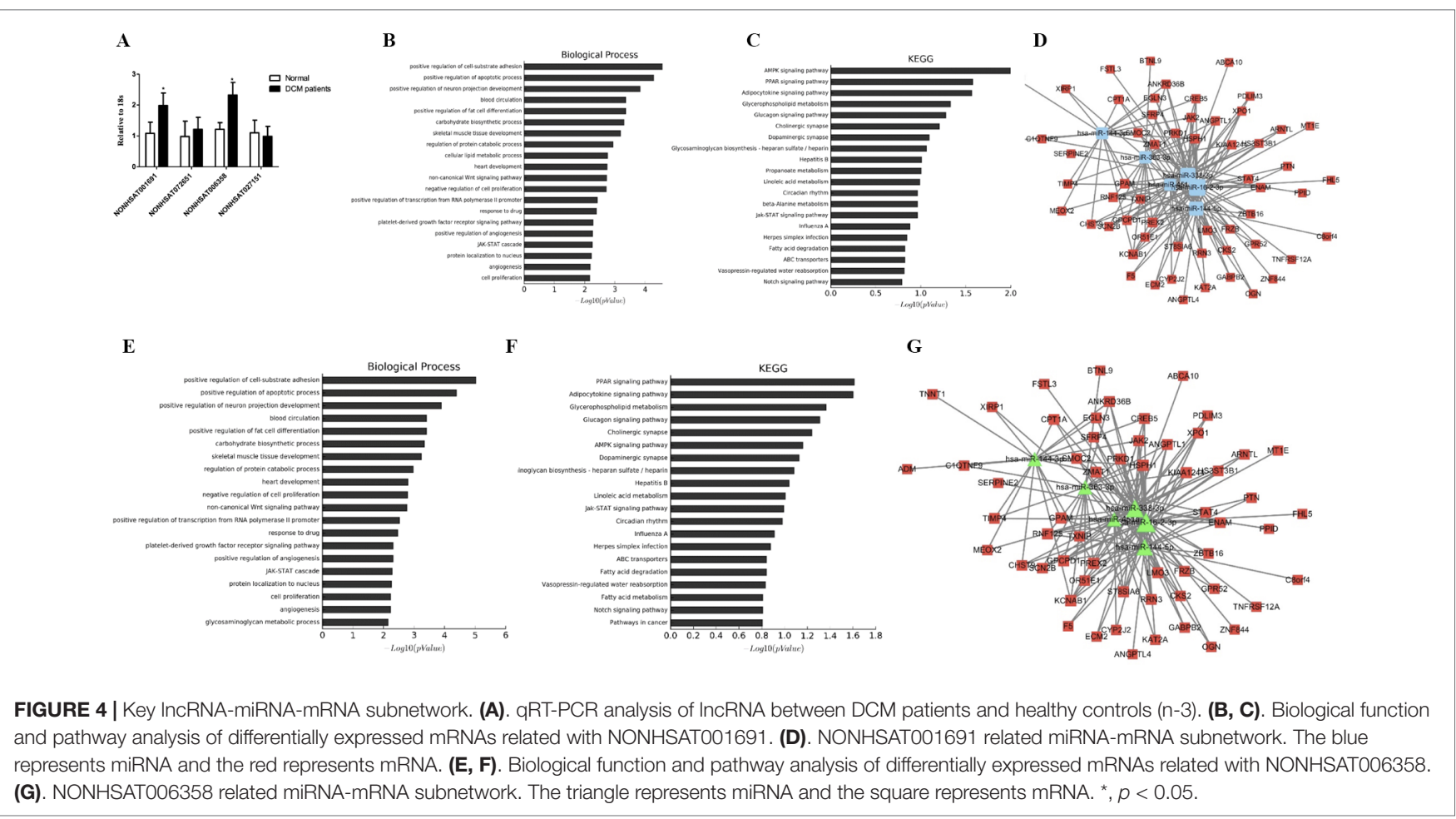



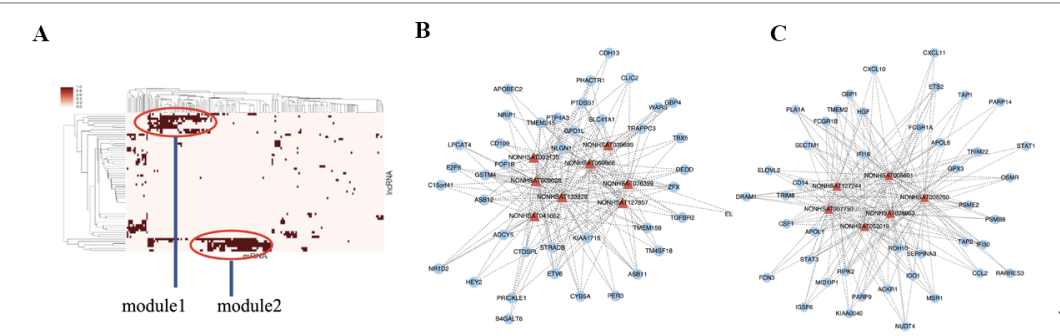

$\mathbf{H}$
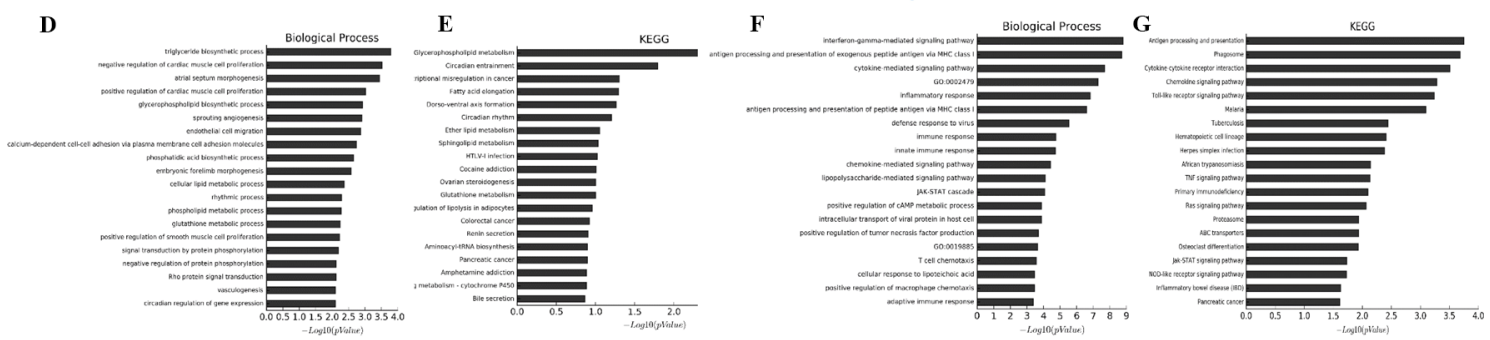

FIGURE 5 | Module analysis of the DCM-related IncRNA-miRNA network. (A). 2 modules were identified highly related to DCM. (B, C). IncRNA-mRNA networks in 2 modules. The triangle represents IncRNA and the circle represents mRNA. (D-G). Biological function and pathway analysis of differentially expressed mRNA related with 2 modules. (H). qRT-PCR analysis of IncRNA between DCM patients and healthy controls $(\mathrm{n}=3) .{ }^{*}, p<0.05$.

versus fibroblasts and demonstrated higher expression level of miR-144-3p and miR-451a in cardiomyocytes, while miR21-5p was enriched in fibroblasts compared to cardiomyocytes (Figure 6A). DCM is characterized by left ventricular dilation and interstitial fibrosis, which are the main causes of heart failure (McNally and Mestroni, 2017). MiR-21-5p was widely expressed in fibroblasts and has been reported to promote transdifferentiation from cardiac fibroblasts into myofibroblasts by targeting notch ligand Jagged1, contributing to cardiac fibrosis post myocardial infarction (Zhou et al., 2018). Then, we placed emphasis on miR-144-3p and miR-451a, which were closed clustered from a single gene locus. miR-451a has been reported to

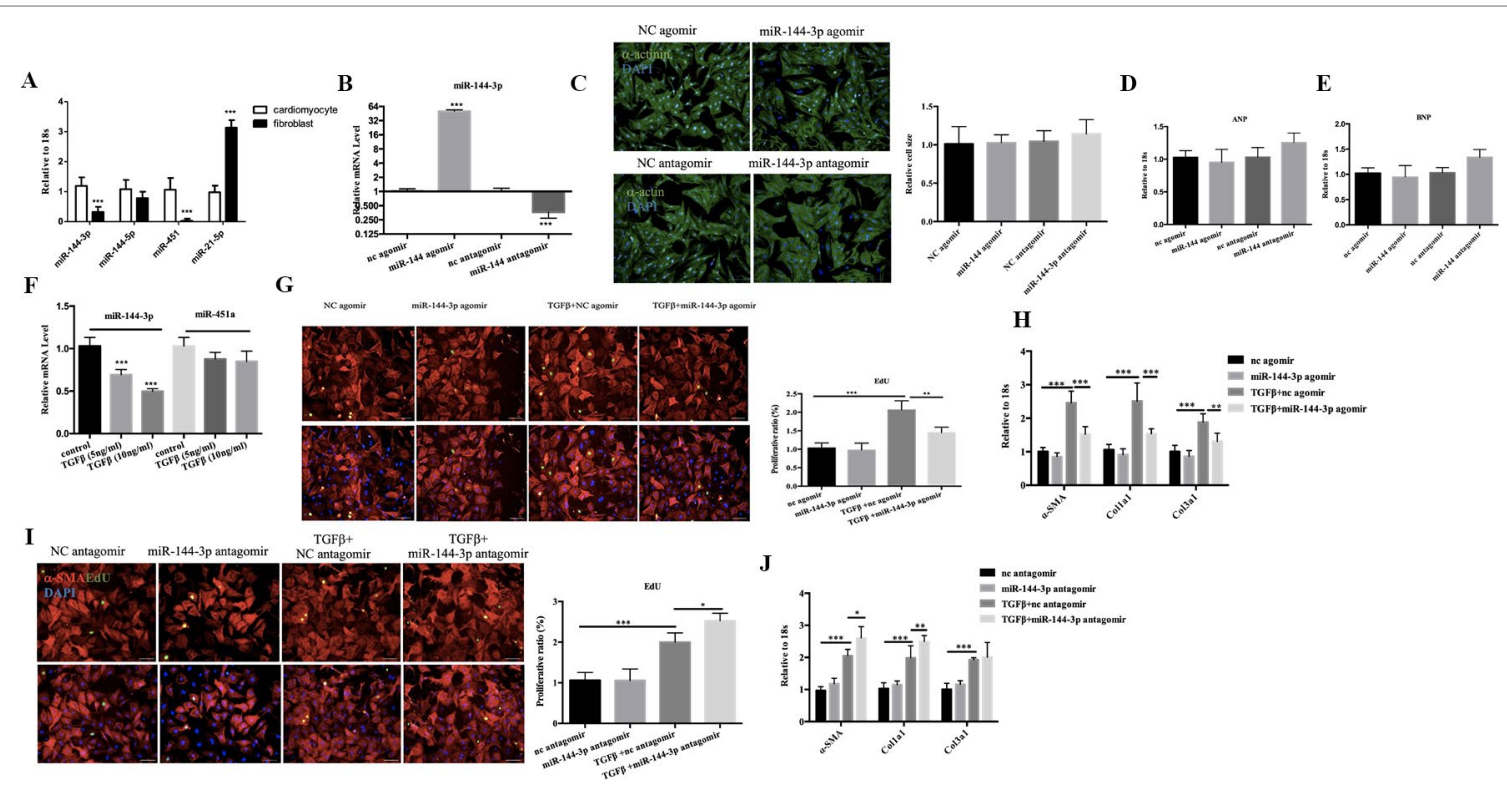

FIGURE 6 | Functional study of miR-144-3p in vitro. (A). qRT-PCR analysis of miRNA between cardiomyocytes and fibroblasts $(n=6)$. (B, C). qRT-PCR analysis of the transfection efficacy of miR-144-3p with agomir/antagomir compared to controls $(n=6)$. (C-E). Overexpression or downregulation of miR-144-3p did not perform effects on cardiomyocyte cell size and pathological hypertrophic markers (ANP and BNP), as evidence by $\alpha$-actin/DAPI staining ( $n=4)$ and qRT-PCR analysis $(n=6)$. (F). Decreased expression of miR-144-3p in cardiac fibrosis model induced by TGF $\beta(n=6)$. (G, H). Forced expression of miR-144-3p attenuated TGF $\beta$-induced cardiac fibroblast proliferation and trans-differentiation, as evidenced by EdU/ $\alpha$-SMA staining $(n=4)$ and qRT-PCR analysis of $\alpha$-SMA, Col1a1 and Col3a1 $(n=6)(\mathbf{I}, \mathbf{J})$. inhibition of miR-144-3p deteriorated TGFb-induced cardiac fibroblasts proliferation and trans-differentiation as evidenced by EdU/ $\alpha$-SMA staining $(\mathrm{n}=4)$ and qRT-PCR analysis $(\mathrm{n}=6)$. Scale bar: 50um. ${ }^{*}, p<0.05 ;{ }^{* \star}, p<0.01 ;{ }^{* \star}, p<0.001$. 
regulate cardiac hypertrophy and autophagy, forced expression of miR-451a in NRCM decreased cell size, whereas knockdown of miR-451a increased cell surface area (Song et al., 2014). Then we transfected miR-144-3p into NRCM and the transfection efficacy of miR-144 agomir or antagomir were exhibited in Figure 6B. However, overexpression or downregulation of miR-144 did not lead to any effects on cell size (Figure 6C) and markers of pathological hypertrophy (Figures 6D, E), supporting a more prominent role for miR-451a in cardiomyocyte hypertrophy rather than miR-144.

Cardiac fibrosis is another hall marker of DCM. By qRTPCR, the expression level of miR-144-3p was identified downregulated in cultured neonatal rat cardiac fibrosis model stimulated by TGF- $\beta$ while miR-451 did not show statistical significance (Figure 6F). To gain mechanistic insight into the role of miR-144 in regulating fibrosis, we investigated the effect of miR-144 in cardiac fibroblasts in vitro. MiR-144-3p overexpression decreased cardiac fibroblasts proliferation and trans-differentiation induced by TGF- $\beta$, as evidenced by a decrease in EdU and $\alpha$-SMA staining, and decreased expression levels of a-SMA, Colla1, and Col3a1(Figures 6G, H). Contrary to the effects of miR-144-3p overexpression, inhibition of miR-144-3p further enhanced cardiac fibroblasts proliferation and differentiation in the presence of TGF- $\beta$ (Figures 6I, J), indicating a potential protective effect of miR-144-3p against cardiac fibrosis.

\section{DISCUSSION}

DCM is an important cause of sudden cardiac death (SCD) and heart failure and is the major indication of cardiac transplantation in children and adults worldwide. DCM is characterized by ventricular chamber enlargement and systolic dysfunction with excessive cardiac fibrosis (McNally and Mestroni, 2017). Over the past few years, great efforts have been made to explore the molecular mechanisms of DCM. miRNA-mediated myocardial gene expression is one of the novel mechanisms in DCM (Naga Prasad and Karnik, 2010; Miyamoto et al., 2015). In this study, we first performed a miRNA microarray using heart samples from DCM patients and healthy controls. miRNAs with a fold change $>$ 2.0 and $P$ value $<0.05$ were further evaluated by qRT-PCR in DCM patients and a doxorubicin-induced cardiomyopathy rodent model. miR-144-3p and miR-451a were identified as down-regulated and miR-21-5p was identified as up-regulated in DCM.

Previous studies have demonstrated that miR-144 and miR-451 are closely clustered and evolutionarily conserved 23 . miR-144 and miR-451 are processed from a single gene locus that is regulated by the essential hematopoietic transcription factor GATA-4 (Zhang et al., 2010). miR-144/451 as a cluster have been identified to play crucial roles in cardiac ischemic lesions. In cardiomyocytes, ectopic expression of miR-144, or miR-451 augmented survival, which was further improved by overexpression of miR-144/451 cluster, compared to controls in response to simulated ischemia/ reperfusion26. In a miR-144/451 knockout mouse model, loss of the miR-144/451 cluster limited the cardio-protection from ischemic preconditioning by up-regulating the Ras-related c3 botulinum toxin substrate 1 (Rac1)-mediated oxidative stress signaling pathway27, indicating a cardio-protective effects of miR-144/451a cluster against ischemic dysfunction. However, little studies have been done regarding the functional role of miR-144/451 on DCM-related pathological changes. Previous studies have shown that forced expression of miR-451a decreased cell size and knockdown of miR451a performed opposite effects (Song et al., 2014). In our study, miR-144-3p did not show any effects on cell size on physiological conditions. Although miR-144-3p was enriched in cardiomyocytes compared to fibroblasts, overexpression of miR-144-3p attenuated fibroblast proliferation and transdifferentiation into myofibroblasts induced by TGF- $\beta$, which is also conforming with previous studies in vivo (He et al., 2018; Li et al., 2018). Therefore, although evolutionarily as a cluster, miR-144-3p and miR-451a may perform distinct roles in DCM. Furthermore, miR-144-3p may also participate in other pathological processes including inflammation $(\mathrm{Hu}$ et al., 2014), autophagy (Li et al., 2018), and mitochondrial metabolism (Li et al., 2016) on cardiomyocytes, which need to be explored in the future.

MiR-21 is one of the first identified miRNAs implicated in cardiac hypertrophy and fibrosis (Duygu and Da Costa Martins, 2015; Zhou et al., 2018). In our study, miR-21-5p was upregulated in DCM heart samples, which was consistent with larger sample study (Satoh et al., 2011). However, the regulatory effects of miR-21-5p on DCM were a result of single cardiac fibrosis or hypertrophy or a combination of diverse pathological process need further exploration.

In addition to miRNAs, accumulated data have shown that lncRNAs participate in a variety of biological processes and complex diseases, including DCM. Unfortunately, functional studies of lncRNAs are relatively more complicated than those of coding RNAs or miRNAs. Therefore, an efficient and accurate way to infer the potential function of lncRNAs is to detect their relationship with miRNAs and/or mRNAs, whose functions have been annotated. In our study, we used the interaction data from NCBI-GEO and our miRNA microarray to generate a global triple network based on the ceRNA theory, which suggests that lncRNAs and mRNAs share the same miRNA in one triplet. The IncRNA-miRNA-mRNA network was composed of 22 lncRNA nodes, 32 mRNA nodes, and 11 miRNA nodes. Then, the hub nodes and the number of relationship pairs were used to perform topological and subnetwork analysis. In general, a lncRNA with more relationship pairs indicates that the lncRNA is a hub that participates in more ceRNA interactions and plays essential roles in network organization. In this study, four lncRNAs (NONHSAT001691, NONHSAT072651, NONHSAT006358, and NONHSAT027151) were observed to be topological key nodes whose node degrees and number of lncRNA-miRNA and miRNA-mRNA pairs were significantly higher than other lncRNAs. These four lncRNAs were then validated in heart samples of DCM patients and healthy controls by using qRT-PCR. NONHSAT001691 and NONHSAT006358 were identified as significantly up-regulated in DCM patients. 
Interestingly, miR-144-3p and miR-451a were the potential targets of these two lncRNAs in the ceRNA network, indicating that the NONHSAT001691/NONHSAT006358-miR-144/451a signaling pathway may play a crucial role in the development of DCM.

GO and pathway analyses have been used to assess biological functions that are enriched among differentially expressed coding genes. Owing to similar miRNA targets, the significant GO terms of NONHSAT001691 and NONHSAT006358 shared common trends involving "positive regulation of cell-substrate adhesion and the apoptotic process," and the results were consistent with those of previous studies on DCM (Miller et al., 2004; Pulinilkunnil et al., 2014; Isserlin et al., 2015). Pathway analysis of NONHSAT001691 and NONHSAT006358 showed that the metabolic pathways were mainly enriched, including the AMPK, PPAR, adipocytokine, glucagon, and fatty acid degradation signaling pathways, all of which have been shown to play important roles in DCM (Nikolaidis et al., 2004; Giannessi et al., 2011; Roh et al., 2014; Sung et al., 2015).

Moreover, bidirectional hierarchical clustering analysis was conducted to investigate the crosstalk between mRNAs and lncRNAs. A total of 10 lncRNAs were found in the ceRNA network, among which four lncRNAs (NONHSAT026953, NONHSAT006250, NONHSAT133928, and NONHSAT041662) were identified as down-regulated in the DCM group by qRTPCR. miR-21-5p was the common target of these four lncRNAs, further confirming the feasibility of our miRNA microarray. Moreover, GO and KEGG analysis of these two modules also indicated that metabolism-related signaling pathways play a crucial role in the development of DCM, which provides a novel direction for the study of mechanisms underlying DCM.

Currently, discovering non-coding RNA-disease associations plays an increasingly vital role in devising diagnostic and therapeutic tools for diseases. However, since uncovering associations via experimental studies are expensive and timeconsuming, novel and effective computational model for the identification of non-coding RNAs associated with DCM or other diseases are in demand. Several different computational methods have been analyzed to calculate potential non-coding RNA (including lncRNAs and miRNAs) -disease association scores (Chen and Yan, 2013; Chen and Huang, 2017; Chen et al., 2017; Chen et al., 2018a; Chen et al., 2018b; Chen et al., 2019). In our study, we constructed a IncRNA-miRNA-mRNA network based on the ceRNA theory. NONHSAT001691/ NONHSAT006358-miR-144-3p/451a and NONHSAT026953/ NONHSAT006250/NONHSAT133928/NONHSAT041662miR-21-5p were further identified as potential key signaling pathways correlated with DCM. Therefore, this study provides the framework of constructing powerful computational model to predict potential lncRNA-miRNA-disease associations and select the most promising DCM or other disease-related lncRNAs/ miRNAs for experimental validation. However, our study still has some limitations. First, owing to the difficulty of obtaining human heart tissues, the sample size was small, totaling six samples (six DCM samples and six healthy control samples). Due to the lack of samples, there may be false positives in the results. Second, in the process of converting different gene IDs from different databases, a number of genes may have been lost, which would decrease the accuracy of our results. Finally, our study was mainly focused on lncRNA/miRNA changes in DCM samples; therefore, the underlying biological functions need further exploration.

\section{DATA AVAILABILITY STATEMENT}

The data on miRNAs discussed in this manuscript have been deposited in NCBI's GEO and are accessible through GEO Series accession number GSE112556.

\section{ETHICS STATEMENT}

The study protocol was approved by the Medical Ethics Committee of the Third Affiliated Hospital of Soochow University in Changzhou, Jiangsu Province, China, and informed consent was obtained from each patient.

\section{AUTHOR CONTRIBUTIONS}

FH led the research team. LT conceived and designed the study. LY and XH developded the methodology. XY analyzed and interpreted the data. LT wrote, reviewed the manuscript.

\section{FUNDING}

This work was supported by grants from the National Natural Science Foundation of China (grant no. 81700343), Natural Science Foundation of Jiangsu Province (grant no. BK20170296), China Postdoctoral Science Foundation (grant no. 2018M642317), PostDoctoral Foundation of Jiangsu Province (grant no. 2018K095B), Six Talent Peaks Project of Jiangsu Province (grant no. WSN-202 to Lichan Tao, grant no. WSW-183 to Fei Hua), Maternal and Child Health Research Project of Jiangsu Province (grant no. F201803), and Changzhou High-Level Medical Talents Training Project (grant no. 2016ZCL J020).

\section{SUPPLEMENTARY MATERIAL}

The Supplementary Material for this article can be found online at: https:/www.frontiersin.org/articles/10.3389/fgene.2019.01149/ full\#supplementary-material

SUPPLEMENTAL TABLE 1 | Clinical and echocardiography parameters for patients with dilated cardiomyopathy.

SUPPLEMENTAL TABLE 2 | qRT-PCR Primers in this study.

SUPPLEMENTAL FIGURE 1 | The echocardiographic parameters including ejection fraction (EF), fraction shortenting (FS), left ventricular mass, left ventricular internal dimension-systole (LVIDs) and left ventricular internal dimension-diastole (LVIDd) for the doxorubicin-induced DCM model. *,$p<0.05$; ${ }^{\star *}, \mathrm{p}<0.01 ;{ }^{* \star *}, \mathrm{p}<0.001$. 


\section{REFERENCES}

Chen, X., and Huang, L. (2017). LRSSLMDA: Laplacian Regularized Sparse Subspace Learning for MiRNA-Disease Association prediction. PloS Comput. Biol. 13, e1005912. doi: 10.1371/journal.pcbi.1005912

Chen, X., Wang, L., Qu, J., Guan, N. N., and Li, J. Q. (2018a). Predicting miRNAdisease association based on inductive matrix completion. Bioinformatics 34, 4256-4265. doi: 10.1093/bioinformatics/bty503

Chen, X., Xie, D., Zhao, Q., and You, Z. H. (2019). MicroRNAs and complex diseases: from experimental results to computational models. Brief. Bioinform. 20, 515-539. doi: 10.1093/bib/bbx130

Chen, X., Yan, C. C., Zhang, X., and You, Z. H. (2017). Long non-coding RNAs and complex diseases: from experimental results to computational models. Brief. Bioinform. 18, 558-576. doi: 10.1093/bib/bbw060

Chen, X., and Yan, G. Y. (2013). Novel human lncRNA-disease association inference based on lncRNA expression profiles. Bioinformatics 29, 2617-2624. doi: 10.1093/bioinformatics/btt426

Chen, X., Yin, J., Qu, J., and Huang, L. (2018b). MDHGI: Matrix Decomposition and Heterogeneous Graph Inference for miRNA-disease association prediction. PloS Comput. Biol. 14, e1006418. doi: 10.1371/journal.pcbi.1006418

Cheng, H. S., Njock, M. S., Khyzha, N., Dang, L. T., and Fish, J. E. (2014). Noncoding RNAs regulate NF-kappaB signaling to modulate blood vessel inflammation. Front.Genet. 5, 422. doi: 10.3389/fgene.2014.00422

Dangwal, S., Schimmel, K., Foinquinos, A., Xiao, K., and Thum, T. (2017). Noncoding RNAs in Heart Failure. Handb. Exp. Pharmacol. 243, 423-445. doi: 10.1007/164_2016_99

Devaux, Y., Zangrando, J., Schroen, B., Creemers, E. E., Pedrazzini, T., Chang, C. P., et al. (2015). Long noncoding RNAs in cardiac development and ageing. Nat. Rev. Cardiol. 12, 415-425. doi: 10.1038/nrcardio.2015.55

Dey, B. K., Mueller, A. C., and Dutta, A. (2014). Long non-coding RNAs as emerging regulators of differentiation, development, and disease. Transcription 5, e944014. doi: 10.4161/21541272.2014.944014

Dobre, D., Borer, J. S., Fox, K., Swedberg, K., Adams, K. F., Cleland, J. G., et al. (2014). Heart rate: a prognostic factor and therapeutic target in chronic heart failure. The distinct roles of drugs with heart rate-lowering properties. Eur. J. Heart Fail 16, 76-85. doi: 10.1093/eurjhf/hft129

Duygu, B., and Da Costa Martins, P. A. (2015). miR-21: a star player in cardiac hypertrophy. Cardiovasc. Res. 105, 235-237. doi: 10.1093/cvr/cvv026

Garin, O., Herdman, M., Vilagut, G., Ferrer, M., Ribera, A., Rajmil, L., et al. (2014). Assessing health-related quality of life in patients with heart failure: a systematic, standardized comparison of available measures. Heart Fail Rev. 19, 359-367. doi: 10.1007/s10741-013-9394-7

Giannessi, D., Caselli, C., Del Ry, S., Maltinti, M., Pardini, S., Turchi, S., et al. (2011). Adiponectin is associated with abnormal lipid profile and coronary microvascular dysfunction in patients with dilated cardiomyopathy without overt heart failure. Metabolism 60, 227-233. doi: 10.1016/j.metabol.2009.12.030

Greco, S., Zaccagnini, G., Perfetti, A., Fuschi, P., Valaperta, R., Voellenkle, C., et al. (2016). Long noncoding RNA dysregulation in ischemic heart failure. J. Transl. Med. 14, 183. doi: 10.1186/s12967-016-0926-5

Haas, J., Mester, S., Lai, A., Frese, K. S., Sedaghat-Hamedani, F., Kayvanpour, E., et al. (2018). Genomic structural variations lead to dysregulation of important coding and non-coding RNA species in dilated cardiomyopathy. EMBO Mol. Med. 10, 107-120. doi: 10.15252/emmm.201707838

He, Q., Wang, F., Honda, T., James, J., Li, J., and Redington, A. (2018). Loss of miR-144 signaling interrupts extracellular matrix remodeling after myocardial infarction leading to worsened cardiac function. Sci. Rep. 8, 16886. doi: 10.1038/s41598-018-35314-6

Hu, Y. W., Hu, Y. R., Zhao, J. Y., Li, S. F., Ma, X., Wu, S. G., et al. (2014). An agomir of miR-144-3p accelerates plaque formation through impairing reverse cholesterol transport and promoting pro-inflammatory cytokine production. PloS One 9, e94997. doi: 10.1371/journal.pone.0094997

Isserlin, R., Merico, D., Wang, D., Vuckovic, D., Bousette, N., Gramolini, A. O., et al. (2015). Systems analysis reveals down-regulation of a network of prosurvival miRNAs drives the apoptotic response in dilated cardiomyopathy. Mol. Biosyst. 11, 239-251. doi: 10.1039/c4mb00265b

Kumarswamy, R., and Thum, T. (2013). Non-coding RNAs in cardiac remodeling and heart failure. Circ. Res. 113, 676-689. doi: 10.1161/ CIRCRESAHA.113.300226
Li, D., Chen, G., Yang, J., Fan, X., Gong, Y., Xu, G., et al. (2013). Transcriptome analysis reveals distinct patterns of long noncoding RNAs in heart and plasma of mice with heart failure. PloS One 8, e77938. doi: 10.1371/journal. pone. 0077938

Li, J., Cai, S. X., He, Q., Zhang, H., Friedberg, D., Wang, F., et al. (2018). Intravenous miR-144 reduces left ventricular remodeling after myocardial infarction. Basic. Res. Cardiol. 113, 36. doi: 10.1007/s003950-018-0694-x

Li, K., Zhang, J., Ji, C., and Wang, L. (2016). MiR-144-3p and Its Target Gene beta-Amyloid Precursor Protein Regulate 1-Methyl-4-Phenyl-1,2-3,6Tetrahydropyridine-Induced Mitochondrial Dysfunction. Mol. Cells 39, 543549. doi: 10.14348 /molcells. 2016.0050

McNally, E. M., and Mestroni, L. (2017). Dilated cardiomyopathy: genetic determinants and mechanisms. Circ. Res. 121, 731-748. doi: 10.1161/ CIRCRESAHA.116.309396

Michalik, K. M., You, X., Manavski, Y., Doddaballapur, A., Zornig, M., Braun, T., et al. (2014). Long noncoding RNA MALAT1 regulates endothelial cell function and vessel growth. Circ. Res. 114, 1389-1397. doi: 10.1161/ CIRCRESAHA.114.303265

Miller, M. K., Granzier, H., Ehler, E., and Gregorio, C. C. (2004). The sensitive giant: the role of titin-based stretch sensing complexes in the heart. Trends. Cell. Biol. 14, 119-126. doi: 10.1016/j.tcb.2004.01.003

Miyamoto, S. D., Karimpour-Fard, A., Peterson, V., Auerbach, S. R., Stenmark, K. R., Stauffer, B. L., et al. (2015). Circulating microRNA as a biomarker for recovery in pediatric dilated cardiomyopathy. J. Heart Lung Transplant. 34, 724-733. doi: 10.1016/j.healun.2015.01.979

Molina-Navarro, M. M., Rosello-Lleti, E., Ortega, A., Tarazon, E., Otero, M., Martinez-Dolz, L., et al. (2013). Differential gene expression of cardiac ion channels in human dilated cardiomyopathy. PloS One 8, e79792. doi: 10.1371/ journal.pone.0079792

Naga Prasad, S. V., and Karnik, S. S. (2010). MicroRNAs-regulators of signaling networks in dilated cardiomyopathy. J. Cardiovasc. Transl. Res. 3, 225-234. doi: 10.1007/s12265-010-9177-7

Nikolaidis, L. A., Elahi, D., Hentosz, T., Doverspike, A., Huerbin, R., Zourelias, L., et al. (2004). Recombinant glucagon-like peptide-1 increases myocardial glucose uptake and improves left ventricular performance in conscious dogs with pacing-induced dilated cardiomyopathy. Circulation 110, 955-961. doi: 10.1161/01.CIR.0000139339.85840.DD

Pinet, F., and Bauters, C. (2015). Potential of non-coding RNA as biomarkers in heart failure. Med. Sci. 31, 770-776. doi: 10.1051/medsci/20153108016

Pourrajab, F., Vakili Zarch, A., Hekmatimoghaddam, S., and Zare-Khormizi, M. R. (2015). The master switchers in the aging of cardiovascular system, reverse senescence by microRNA signatures; as highly conserved molecules. Prog. Biophys. Mol. Biol. 119, 111-128. doi: 10.1016/j.pbiomolbio.2015.05.004

Pulinilkunnil, T., Kienesberger, P. C., Nagendran, J., Sharma, N., Young, M. E., and Dyck, J. R. (2014). Cardiac-specific adipose triglyceride lipase overexpression protects from cardiac steatosis and dilated cardiomyopathy following dietinduced obesity. Int. J. Obes. 38, 205-215. doi: 10.1038/ijo.2013.103

Roh, J. I., Cheong, C., Sung, Y. H., Lee, J., Oh, J., Lee, B. S., et al. (2014). Perturbation of NCOA6 leads to dilated cardiomyopathy. Cell. Rep. 8, 991-998. doi: 10.1016/j.celrep.2014.07.027

Satoh, M., Minami, Y., Takahashi, Y., Tabuchi, T., and Nakamura, M. (2011). A cellular microRNA, let-7i, is a novel biomarker for clinical outcome in patients with dilated cardiomyopathy. J. Card. Fail. 17, 923-929. doi: 10.101/j. cardfail.2011.07.012

Schiano, C., Costa, V., Aprile, M., Grimaldi, V., Maiello, C., Esposito, R., et al. (2017). Heart failure: Pilot transcriptomic analysis of cardiac tissue by RNAsequencing. Cardiol. J. 24, 539-553. doi: 10.5603/CJ.a2017.0052

Sen, R., Ghosal, S., Das, S., Balti, S., and Chakrabarti, J. (2014). Competing endogenous RNA: the key to posttranscriptional regulation. Sci. World J. 2014, 896206. doi: 10.1155/2014/896206

Shi, X., Sun, M., Wu, Y., Yao, Y., Liu, H., Wu, G., et al. (2015). Post-transcriptional regulation of long noncoding RNAs in cancer. Tumour. Biol. 36, 503-513. doi: 10.1007/s13277-015-3106-y

Song, L., Su, M., Wang, S., Zou, Y., Wang, X., Wang, Y., et al. (2014). MiR-451 is decreased in hypertrophic cardiomyopathy and regulates autophagy by targeting TSC1. J. Cell. Mol. Med. 18, 2266-2274. doi: 10.1111/jcmm.12380

Sung, M. M., Zordoky, B. N., Bujak, A. L., Lally, J. S., Fung, D., Young, M. E., et al. (2015). AMPK deficiency in cardiac muscle results in dilated cardiomyopathy 
in the absence of changes in energy metabolism. Cardiovasc. Res. 107, 235-245. doi: $10.1093 / \mathrm{cvr} / \mathrm{cvv} 166$

Tao, L., Bei, Y., Chen, P., Lei, Z., Fu, S., Zhang, H., et al. (2016). Crucial Role of miR-433 in Regulating Cardiac Fibrosis. Theranostics 6, 2068-2083. doi: 10.7150/thno. 15007

Tao, L., Bei, Y., Li, Y., and Xiao, J. (2018). Neonatal rat cardiomyocytes isolation, culture, and determination of microRNAs' effects in proliferation. Methods Mol. Biol. 1733, 203-213. doi: 10.1007/978-1-4939-7601-0_17

Tay, Y., Rinn, J., and Pandolfi, P. P. (2014). The multilayered complexity of ceRNA crosstalk and competition. Nature 505, 344-352. doi: 10.1038/ nature 12986

Thum, T. (2015). Facts and updates about cardiovascular non-coding RNAs in heart failure. ESC. Heart. Fail. 2, 108-111. doi: 10.1002/ehf2.12054

Uchida, S., and Dimmeler, S. (2015). Long noncoding RNAs in cardiovascular diseases. Circ. Res. 116, 737-750. doi: 10.1161/CIRCRESAHA

Zhang, X., Wang, X., Zhu, H., Zhu, C., Wang, Y., Pu, W. T., et al. (2010). Synergistic effects of the GATA-4-mediated miR-144/451 cluster in protection against simulated ischemia/reperfusion-induced cardiomyocyte death. J. Mol. Cell. Cardiol. 49, 841-850. doi: 10.1016/j.yjmcc.2010.08.007

Zhou, X. L., Xu, H., Liu, Z. B., Wu, Q. C., Zhu, R. R., and Liu, J. C. (2018). miR-21 promotes cardiac fibroblast-to-myofibroblast transformation and myocardial fibrosis by targeting Jagged1. J. Cell. Mol. Med. 22, 3816-3824. doi: 10.1111/ jcmm.13654

Conflict of Interest: The authors declare that the research was conducted in the absence of any commercial or financial relationships that could be construed as a potential conflict of interest.

Copyright $\odot 2019$ Tao, Yang, Huang, Hua and Yang. This is an open-access article distributed under the terms of the Creative Commons Attribution License (CC $B Y)$. The use, distribution or reproduction in other forums is permitted, provided the original author(s) and the copyright owner(s) are credited and that the original publication in this journal is cited, in accordance with accepted academic practice. No use, distribution or reproduction is permitted which does not comply with these terms. 